\title{
Liquid Biopsy in Breast Cancer
}

\author{
Lorena Incorvaia, Marta Castiglia, \\ Alessandro Perez, Daniela Massihnia, \\ Stefano Caruso, Sevilay Altintas, Valentina Calò, \\ and Antonio Russo
}

\section{Introduction}

Breast cancer $(\mathrm{BC})$ to date remains the most common cancer in women [1].

The increased incidence is due to wide introduction of mammography screening programs and continues to grow with the aging of the population, while the prevalence is increasing as a consequence of improvements in treatment outcomes. At the same time, mortality has decreased thanks to an efficient screening that enables disease diagnosis at a very early stage. Moreover, chemotherapy and endocrine adjuvant therapy have strongly implemented treatment in BC.

Lorena Incorvaia and Marta Castiglia contributed equally to this work.

L. Incorvaia $\bullet$ M. Castiglia $\bullet$ A. Perez $\bullet$ D. Massihnia

V. Calò • A. Russo, MD, PhD ( $\varangle)$

Department of Surgical, Oncological and Oral

Sciences, Section of Medical Oncology, University

of Palermo, Via del Vespro 129, 90127 Palermo, Italy

e-mail: lorena.incorvaia@unipa.it;

martacastiglia@gmail.com; ale-like@libero.it; danielamassi87@gmail.com;

valentinacalo74@libero.it; antonio.russo@usa.net

S. Caruso, MD, $\mathrm{PhD}(\bowtie)$

Génomique Fonctionnelle des Tumeurs Solides, INSERM, UMR 1162, 75010 Paris, France e-mail: steno.caruso@gmail.com

S. Altintas

Multidisciplinary Oncologic Centre Antwerp

(MOCA), Edegem, Belgium
Nowadays, BC is often diagnosed at local disease stage, and, after surgery, based on individual's risk of relapse, the patients undergo adjuvant systemic treatment or/and regional irradiation to decrease the risk of recurrence. Some patients, however, will eventually develop recurrent or metastatic disease.

According to standard practice, the choice of treatment strategy includes assays for estrogen (ER) and progesterone (PgR) receptor expression levels, overexpression of human epidermal growth factor receptor 2 (Her-2), or amplification status of the correlate oncogene, but also histological grade and Ki67 to evaluate proliferation of tumor cells.

These features result in the identification of different clinical subgroups of BC:

- The "luminal" tumors, which express ER and PgR receptors and are characterized by endocrine responsiveness and further subdivided into "luminal A" and "luminal B" according to the expression levels of Ki67

- The "Her-2 positive" subgroup, which gets clinical benefit from treatment with "trastuzumab," selective monoclonal antibody that targets Her-2, used in both early and advanced disease settings

- The "triple-negative" subgroup, characterized by the absence of the tree receptors, hormonal receptors, and Her-2, with, therefore, a lower availability of therapeutic options 
On the basis of new molecular diagnostic techniques of genomic profiling, today we know that to each clinical subgroup of BC corresponds a specific molecular subtype with distinct genomic signatures, conditioning the biologic behavior of tumors [2-4].

Current BC classification and assessment remain strongly based on clinicopathological criteria, including patient age, tumor size, lymph node invasion, histological type, and grade.

Nevertheless the established clinicopathological parameters are not sufficient anymore for risk stratification and clinical decision-making, particularly regarding adjuvant chemotherapy, since substantial over- or undertreatment may occur. $\mathrm{ER}, \mathrm{PgR}$, and Her-2 status, used for many years as only validated predictive factors to select patients for endocrine treatment and anti-Her-2 treatments, provide limited information.

Thus, novel molecular markers are under investigation to achieve a more precise prognostic and predictive evaluation of disease and a more effective "personalized treatment" in BC.

Clinicopathological information should be combined with genomic profiling to estimate recurrence risk and identify high-risk BC patients (prognostic value) and predict optimal treatment for each disease subgroup (predictive value).

\section{Reading the Breast Cancer Genome: An Explosion of Biomarker Diversity}

The recent introduction of translational analysis techniques, mainly next-generation sequencing (NGS), has led to an enormous genomic data about BC that helped the identification of several molecular alterations associated with the distinct molecular subtype of BC.

This information has revealed that $\mathrm{BC}$ is not a single disease but a complex and heterogeneous tumor, complicating our understanding toward molecular makeup of the tumor.

Over the tumor heterogeneity from different individuals (intertumor heterogeneity), even with the same clinicopathological features, there is a spatial intra-tumor heterogeneity due to subpopulations of tumor cells with different genomic alterations coexisting within the same tumor and a temporal intra-tumor heterogeneity of different cells in the same patients but at different time points, for example, between primary tumor and its metastasis (Fig. 9.1) [5-7].

This phenomenon represents one of the main barriers to precision medicine in breast cancer: the information obtained from standard tumor tissue sampling cannot be the same for the whole tumor and offer a static picture of disease. The constant molecular change of tumor cell population, spatial and temporal, requires a noninvasive approach, for real-time picture of disease. Liquid biopsy is a useful tool to follow the continuously evolving genomic landscape of breast cancer [810] (Fig. 9.2).

\section{Circulating Tumor DNA (ctDNA)}

Several studies have shown that ctDNA can be used in clinical practice for evaluation and decision-making in the diagnosis, treatment, and follow-up of breast cancer patients [11]. Indeed, it has been demonstrated that high levels of ctDNA correlate with tumor size, lymph node involvement, histopathological grade, and clinical staging $[11,12]$. ctDNA is easier to detect in patients with metastatic breast cancer compared to patients that have a localized disease and concentrations of ctDNA increased with advanced stage of cancer [13].

Different researchers have focused on mutational analysis of genes directly involved in breast cancer both in patients with an advancedstage disease and in patients with localized disease. Some studies have quantified the presence of tumor-specific alterations in ctDNA. In the screening and diagnosis of breast cancer, patientspecific mutations are not known before. Therefore, these studies have focused on cancerassociated alterations that are common in all types of breast cancers. Chimonidou et al. found CST6 promoter methylation in plasma ctDNA in $13-40 \%$ of breast cancer patients but none in healthy patients [14]. Accordingly, Dulaimi et al. found hypermethylation of promoters RASSF1A, APS, and DAP kinase in the serum of $70 \%$ breast cancer patients and none in serum from healthy subjects [15]. Oshiro et al. developed a digital 
Fig. 9.1 Tumor heterogeneity in breast cancer: (a) intertumor heterogeneity, (b) spatial intra-tumor heterogeneity, and (c) temporal intra-tumor heterogeneity
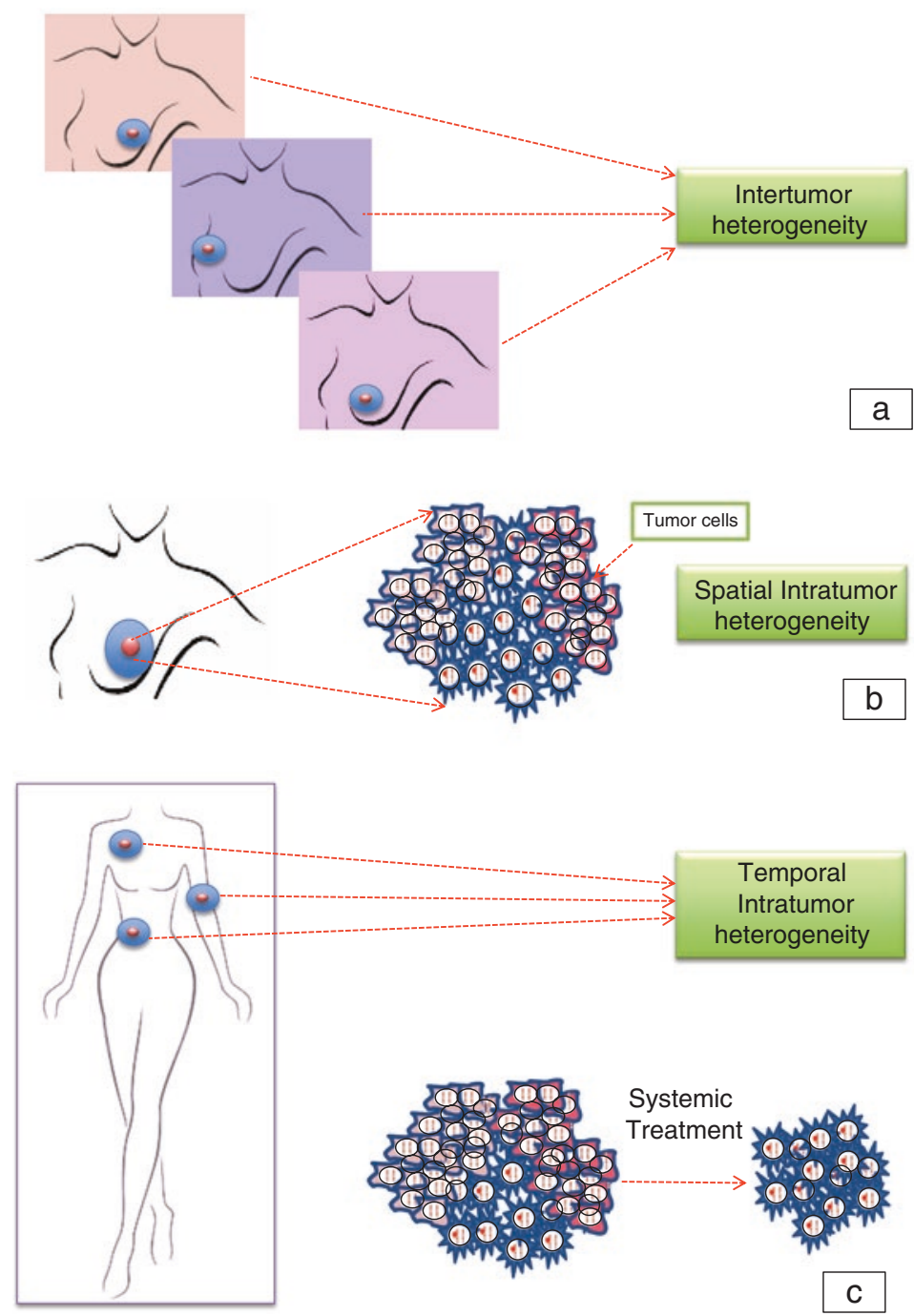

PCR assay to evaluate three hotspot PIK3CA mutations, which is one of the main gene involved in breast cancer tumorigenesis [3]. Again by comparing healthy women with stage I-III breast cancer patients, it was shown that PIK3CA mutations in ctDNA were only detectable in the latter group [16] with a frequency of $23 \%$ (Fig. 9.3). Interestingly, Board et al. detected PIK3CA mutations in ctDNA in $80 \%$ of patients with metastatic cancer, demonstrating that advanced patients have more circulating DNA [17]. In another study, it showed that ctDNA was detectable in $86 \%$ of patients with advanced breast cancer but only $50 \%$ of patients with localized disease and at early stage [18].
Some studies have used baseline ctDNA levels to predict patients' prognosis, but results obtained are contradictory. Iqbal et al. performed a comprehensive analysis of circulating cell-free DNA in serum by the evaluation of DNA integrity index. To this end, qPCR analysis of Alu sequencing using fragments of $115 \mathrm{bp}$ and 247 bp was performed in 148 BC patients at baseline, 47 patients postoperative, and 51 healthy controls. They showed that DNA integrity was significantly higher in stage IV than earlier stages, and it decreases after surgery. Moreover, DNA integrity was able to stratify patients in two groups, relapsed and disease-free patients, with higher DNA integrity in relapsed 


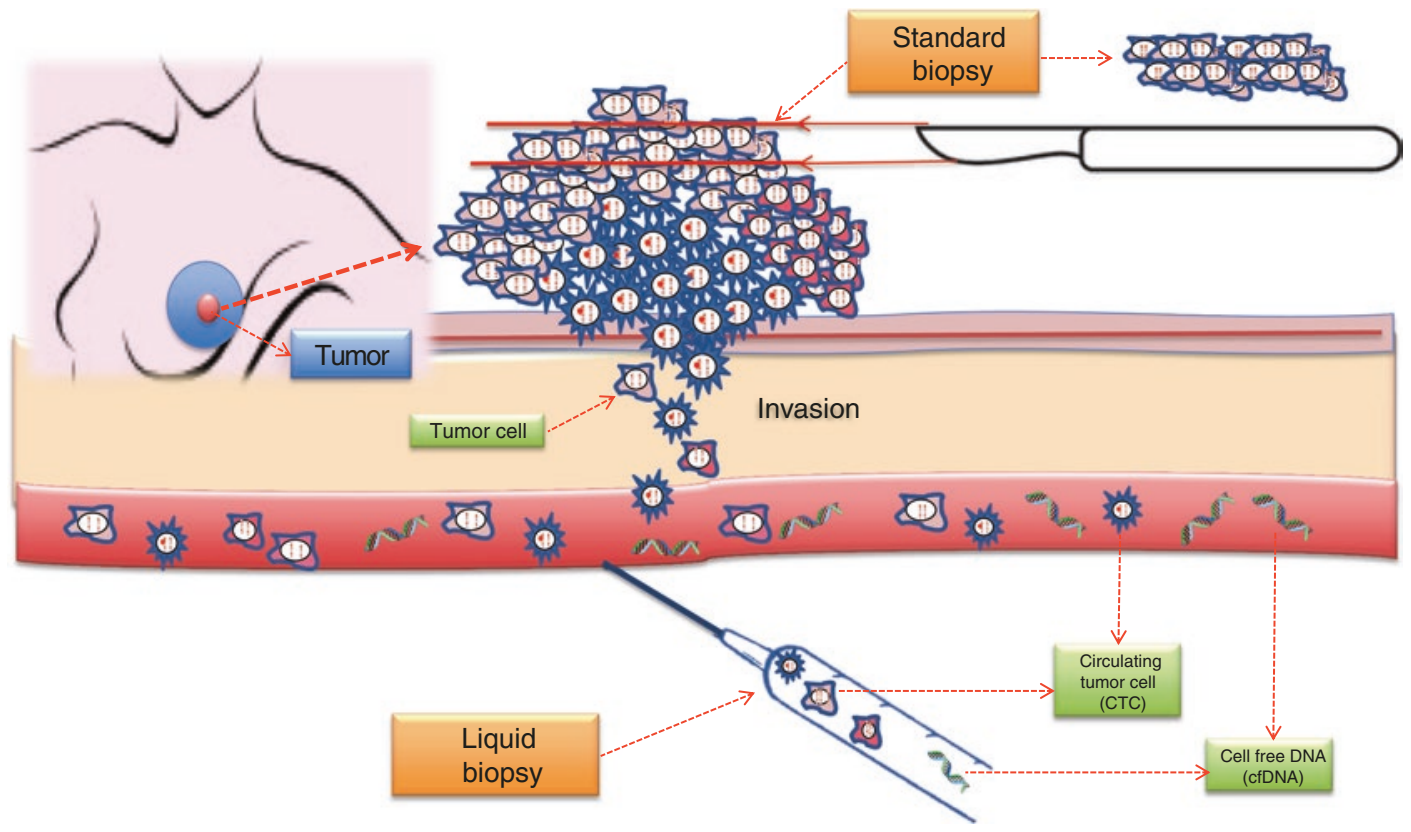

Fig.9.2 Standard biopsy and liquid biopsy in breast cancer: the differences for a "picture" of disease

Fig.9.3 Schematic representation of the Oshiro study design

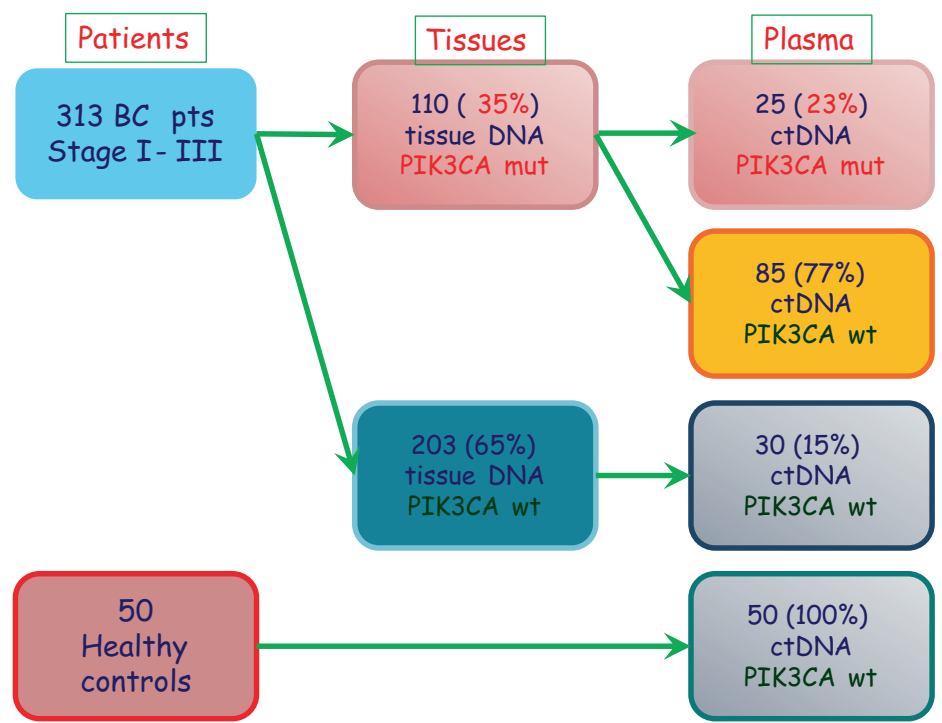

ctDNA may also be used to monitor treatment efficacy. Recent studies in breast cancer patients have found a decrease in ctDNA concentrations after surgery and chemotherapy. This prompted further studies into the use of ctDNA as a marker of treatment response [22]. Dawson et al. have compared ctDNA and CTCs for the monitoring of response to therapy in metastatic breast cancer patients. In this study, somatic mutations and 
structural variants were first analyzed in tumor tissue and then confirmed in plasma samples using both a microfluidic digital PCR assay and sequencing. ctDNA was detected in 29 of 30 women in 115 of 141 plasma samples collected during 2 years' time. Fluctuations in ctDNA correlate with treatment responses as also confirmed by imaging analysis. For 19 women who had progressive disease on CT imaging, 17 had growing levels of ctDNA, whereas only 7 had also CTC increase. In 10 of the 19 patients with progression, ctDNA increased an average of 5 months before the establishment of progressive disease on imaging. Increasing levels of both ctDNA and CTCs were associated with inferior OS. This group have found that ctDNA have a superior sensitivity and improved correlation with changes in tumor burden, promoting a better measure of treatment effectiveness for metastatic patients [23].

ctDNA can also be used to investigate tumor heterogeneity and clonal evolution. It is known in the literature that the metastatic cancer has different characteristics than the primary tumor [24]. Primary tumor biopsies cannot follow the evolutionary changes between metastatic lesions and primary tumor [25]. Despite these data, the current treatment decisions are often based on the molecular profile of the primary tumor without taking into consideration the heterogeneity of metastatic cancer. Moreover, many patients refuse a second tissue biopsy because the technique is very invasive and painful. Given that the ctDNA is released from all tumor components, it may provide a more complete molecular profile of changing subclone populations and better guide therapy [26]. De Mattos-Arruda et al. examined primitive tumor DNA, liver metastasis DNA, and ctDNA collected from plasma at different time points in one patient with ER+/HER2 invasive ductal lobular carcinoma with liver metastasis. They identified 16 mutations in the liver metastasis, and only 9 were also detectable in the primary tumor. Thus, ctDNA may provide a more complete picture of the mutational landscape of metastatic disease.

Based on the previously mentioned studies, it can be stated that the ctDNA could be become a valid biomarker with applications from diagnosis to prognosis but also for the monitoring of tumor evolution and therapy response, but numerous studies are still needed to go all in one direction.

\section{Minimal Residual Disease (MRD)}

Nowadays, one of the main attempts in breast cancer management is testing the feasibility of liquid biopsies to evaluate the minimal residual disease (MRD). The term MRD can be defined as the lowest levels of residual disease after a curative approach either surgical or pharmacological. In fact, evidence of MRD after first-line treatment may be clinically useful to decide whether an adjuvant treatment is requested in order to avoid any possibility of disease recurrence [27].

In the perspective of a painless and noninvasive monitoring of the disease over time, liquid biopsies can be easily used as a feasible tool to monitor MRD also in breast cancer. In particular, MRD represents a higher challenging clinical condition in early-stage tumors (nonmetastatic), while the spread of circulating biomarkers (ctDNA, CTCs) from primary tumor is still not massive. To date, big efforts are still needed to identify which patients, among those who underwent to curative surgery, are completely diseasefree from those who still present hidden residual disease that causes relapse. Moreover, a proper evaluation of MRD could spare disease-free patients from receiving useless but still aggressive adjuvant chemotherapy [28]. Therefore, the detection of ctDNA prior and after surgery and/or radiotherapeutic intervention would be fundamental in predicting residual disease [29]. In 2014, Beaver and its group attempted for the first time to highlight the use of liquid biopsy for stratifying patients on the basis of the risk of recurrence in a relatively small cohort of 30 early-stage breast cancer patients. Indeed, by using droplet digital PCR (ddPCR), PIK3CA exon 9 and 20 mutations have been assessed in primary breast tumors and paired pre- and postsurgery plasma samples of ER+/PR+ early-stage breast cancer patients (Fig. 9.4). The presurgery tissue samples have been firstly analyzed by Sanger sequencing for PIK3CA mutations and then confirmed by ddPCR. The digital approach showed five more patients (15/30) positive for 
PI3Ks: a family of lipid kinases grouped in 3 classes (PIP2 to PIP3)

$\square$ Class IA PI3Ks: heterodimeric proteins $\left\{\begin{array}{l}\text { Regulatory (p85) subunit } \\ \text { Catalytic (p110) subunit }\end{array}\right.$

$\square$ PIK3CA (encoded p110a): proto-oncogene, chromosome 3 (3q26.3), 20 encoding exons

$\square$ PIK3CA mutations in BC: $35-40 \%$

$\square$ Hot-spot of mutation: Exon $9(60-65 \%)$ and Exon 20 (20-25\%)

Mutations in exon 9 PIK3CA co-existed with RAS and BRAF mutation

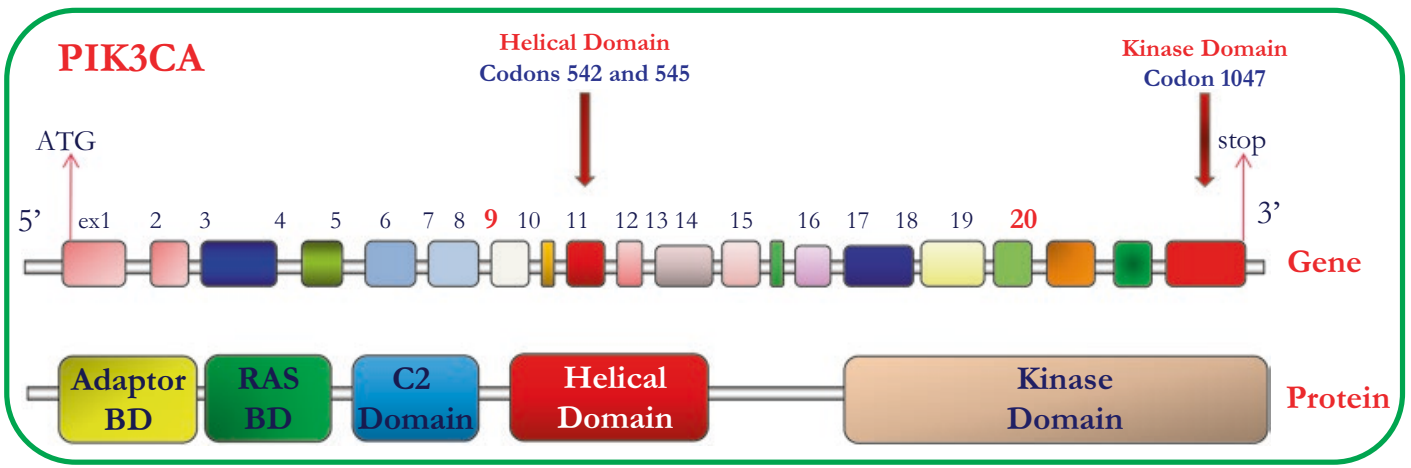

Fig. 9.4 Molecular and biochemical characteristics of PIK3CA domains

PIK3CA mutation with respect to the previous approach. Circulating plasma DNA has been then extracted from pre- and postsurgery blood samples. PIK3CA mutational analysis through ddPCR on presurgery plasma samples showed that of the 15 PIK3CA mutations previously detected in FFPE samples, 14 mutations have been also found in the paired plasma samples with high sensitivity (93.3\%) and specificity $(100 \%)$. Postsurgery plasma samples have been collected, at times ranging from 15 to 72 days after surgery, from 10/15 patients with PIK3CA mutations detected in plasma DNA before surgery. Indeed, five patients had detectable ctDNA demonstrating a still-residual disease despite any clinical or radiological evidence of disease [30]. More recently, Garcia-Murillas et al. have traced PIK3CA mutation in plasma samples to predict relapse in early-stage tumors. In this prospective study, the ddPCR analysis of 55 plasma samples of early breast cancer patients under neoadjuvant chemotherapy was able to anticipate almost
8 months the clinical evidence of metastatic relapse [31]. In 2016, the group of Riva et al. focused on the feasibility of liquid biopsy for the detection of MRD in a cohort of nonmetastatic TBNC patients during neoadjuvant chemotherapy (NCT). Plasma samples were collected at four different time points: before NCT, after one cycle, presurgery, and postsurgery for $36 / 40$ TNBC patients. The analysis of ctDNA has been performed through ddPCR, analyzing TP53 mutations, one of the most common genetic alterations in TNBC. ddPCR analysis showed that before NCT, ctDNA was detected in 27/36 patients, and its levels were significantly correlated with tumor size, tumor stage, as well as mitotic index. After the first NCT cycle, a remarkable decrease of ctDNA levels has been showed for all patients except for one who instead showed increased ctDNA levels. Interestingly, this patient experienced disease progression during chemotherapy. Furthermore, no patients showed detectable ctDNA after surgery [32]. Therefore, liquid 
biopsy seems to represent a valuable option in the management and monitoring of breast cancer patients. In particular, in minimal residual disease, the detectability of circulating biomarkers in early-stage disease would open thus the possibility to enroll these patients in specific surveillance programs and consequently get future benefits through longer-term follow-up.

\section{Circulating Tumor Cells (CTCs)}

The hematogenous spread of single tumor cells from the primary tumor was first demonstrated in the nineteenth century. In the beginning, the aim was to investigate disseminated tumor cells (DTCs) in the bone marrow. Indeed, in 2005, it was first published a multicenter pooled metaanalysis that assessed the prognostic significance of DTCs in the bone marrow at the time of diagnosis. In particular, the study included 4703 patients diagnosed with stage I, II, and III breast cancer and followed over a 10-year follow-up period. This study highlighted for the first time that patients with bone marrow micrometastasis have larger tumors and tumors with a higher histological grade. Moreover, those patients have lymph node metastasis and hormone-receptor negative tumors. The presence of micrometastasis was a significant prognostic factor with respect to poor overall survival and breast cancerspecific survival (univariate mortality ratios, 2.15 and 2.44, respectively; $p<0.001$ for both outcomes) and poor disease-free survival and distant-disease-free survival during the 10-year observation period (incidence rate ratios, 2.13 and 2.33, respectively; $p<0.001$ for both outcomes) (reference).

Nowadays, big efforts are still needed to improve the molecular characterization of a highly heterogeneous tumor. Indeed, studying CTCs would be helpful to improve clinical outcome in particular in triple-negative breast cancer (TNBC). Recently, Angelaki et al. studied CTC phenotype in a cohort including early-stage and metastatic TNBC and hormone-positive breast cancers before and after adjuvant chemotherapy. Expression of ER, PR, CK, HER2, and EGFR on
CTCs has been assessed through immunochemistry. In early-stage TNBC, before any adjuvant chemotherapy, the predominant CTC phenotypes were $\mathrm{ER}+(24.4 \%), \mathrm{PR}+(24.4 \%), \mathrm{CK}+/ \mathrm{HER} 2+$ (20\%), and CK+/EGFR+ (40\%). Moreover, in early-stage TNBC, a high risk of relapse is correlated with the $\mathrm{CK}+\mathrm{HR}$ - phenotype, and, in particular, the $\mathrm{CK}+\mathrm{PR}$ - phenotype is often accompanied by decreased DFI $(p=0.04)$ and OS $(p=0.032)$, demonstrating that these cells may have an aggressive metastatic potential. This study also focused on characterizing CTC subpopulation after adjuvant treatment. Indeed, immunochemistry showed a decreased isolation of HER2-positive CTCs in comparison to ER/PR CTCs. In fact, we can speculate that chemotherapy does not have the same efficacy against all CTC subpopulations. Otherwise, in metastatic cancer, the incidence of CK+/HER2+ CTCs was higher than the early-stage counterpart. Indeed, this finding can predict a more aggressive behavior during disease evolution [33]. The prognostic value of CTC count with respect to the most known unfavorable prognostic factors as progression-free survival (PFS) and overall survivor (OS) has been deeply evaluated in breast cancer. In the study from Bidard et al., CTC count has been evaluated through the CellSearch method before starting a new treatment and after 3-5 and 6-8 weeks after the treatment in a cohort of 2400 patients recruited among 19 different centers. In fact, they demonstrated that a number of five CTCs per $7.5 \mathrm{~mL}$ or higher are often associated with decreased PFS and OS if compared with patients with a number of CTCs less than 5 per $7.5 \mathrm{~mL}$. Moreover, increased CTC number both at time 3-5 and 6-8 weeks after the new treatment is significantly correlated with shortened PFS and OS and overall to poorer prognosis [34].

\section{References}

1. Ferlay J, Soerjomataram I, Dikshit R, et al. Cancer incidence and mortality worldwide: sources, methods and major patterns in GLOBOCAN 2012. Int J Cancer. 2015;136(5):E359-86.

2. Reis-Filho JS, Pusztai L. Gene expression profiling in breast cancer: classification, prognostication, and prediction. Lancet. 2011;378(9805):1812-23. 
3. Network CGA. Comprehensive molecular portraits of human breast tumours. Nature. 2012;490(7418):61-70.

4. Eroles P, Bosch A, Pérez-Fidalgo JA, Lluch A. Molecular biology in breast cancer: intrinsic subtypes and signaling pathways. Cancer Treat Rev. 2012;38(6):698-707.

5. Bedard PL, Hansen AR, Ratain MJ, Siu LL. Tumour heterogeneity in the clinic. Nature. 2013;501(7467): 355-64.

6. Burrell RA, McGranahan N, Bartek J, Swanton C. The causes and consequences of genetic heterogeneity in cancer evolution. Nature. 2013;501(7467): 338-45.

7. Curtit E, Nerich V, Mansi L, et al. Discordances in estrogen receptor status, progesterone receptor status, and HER 2 status between primary breast cancer and metastasis. Oncologist. 2013;18(6):667-74.

8. De Mattos-Arruda L, Weigelt B, Cortes J, et al. Capturing intra-tumor genetic heterogeneity by de novo mutation profiling of circulating cell-free tumor DNA: a proof-of-principle. Ann Oncol. 2014;25(9): 1729-35.

9. De Mattos-Arruda L, Caldas C. Cell-free circulating tumour DNA as a liquid biopsy in breast cancer. Mol Oncol. 2016;10(3):464-74.

10. Diehl F, Schmidt K, Choti MA, et al. Circulating mutant DNA to assess tumor dynamics. Nat Med. 2008;14(9):985-90.

11. Loman N, Saal LH. The state of the art in prediction of breast cancer relapse using cell-free circulating tumor DNA liquid biopsies. Ann Transl Med. 2016;4(Suppl 1):S68.

12. Catarino R, Ferreira MM, Rodrigues $H$, et al. Quantification of free circulating tumor DNA as a diagnostic marker for breast cancer. DNA Cell Biol. 2008;27(8):415-21.

13. Canzoniero JV, Park BH. Use of cell free DNA in breast oncology. Biochim Biophys Acta. 2016; 1865(2):266-74.

14. Chimonidou M, Tzitzira A, Strati A, et al. CST6 promoter methylation in circulating cell-free DNA of breast cancer patients. Clin Biochem. 2013;46(3): 235-40.

15. Dulaimi E, Hillinck J. Ibanez de Caceres I, Al-Saleem T, cairns P. Tumor suppressor gene promoter hypermethylation in serum of breast cancer patients. Clin Cancer Res. 2004;10(18 Pt 1):6189-93.

16. Oshiro C, Kagara N, Naoi Y, et al. PIK3CA mutations in serum DNA are predictive of recurrence in primary breast cancer patients. Breast Cancer Res Treat. 2015;150(2):299-307.

17. Board RE, Wardley AM, Dixon JM, et al. Detection of PIK3CA mutations in circulating free DNA in patients with breast cancer. Breast Cancer Res Treat. 2010;120(2):461-7.

18. Bettegowda C, Sausen M, Leary RJ, et al. Detection of circulating tumor DNA in early- and late-stage human malignancies. Sci Transl Med. 2014;6(224):224ra224.
19. Iqbal S, Vishnubhatla S, Raina V, et al. Circulating cell-free DNA and its integrity as a prognostic marker for breast cancer. Springerplus. 2015;4:265.

20. Bechmann T, Andersen RF, Pallisgaard N, et al. Plasma HER2 amplification in cell-free DNA during neoadjuvant chemotherapy in breast cancer. J Cancer Res Clin Oncol. 2013;139(6):995-1003.

21. Madic J, Kiialainen A, Bidard FC, et al. Circulating tumor DNA and circulating tumor cells in metastatic triple negative breast cancer patients. Int J Cancer. 2015;136(9):2158-65.

22. Forshew T, Murtaza M, Parkinson C, et al. Noninvasive identification and monitoring of cancer mutations by targeted deep sequencing of plasma DNA. Sci Transl Med. 2012;4(136):136ra168.

23. Dawson SJ, Tsui DW, Murtaza M, et al. Analysis of circulating tumor DNA to monitor metastatic breast cancer. N Engl J Med. 2013;368(13):1199-209.

24. Arnedos M, Vicier C, Loi S, et al. Precision medicine for metastatic breast cancer--limitations and solutions. Nat Rev Clin Oncol. 2015;12(12):693-704.

25. Martelotto LG, Ng CK, Piscuoglio S, Weigelt B, ReisFilho JS. Breast cancer intra-tumor heterogeneity. Breast Cancer Res. 2014;16(3):210.

26. Aparicio S, Caldas C. The implications of clonal genome evolution for cancer medicine. N Engl J Med. 2013;368(9):842-51.

27. Tachtsidis A, McInnes LM, Jacobsen N, Thompson EW, Saunders CM. Minimal residual disease in breast cancer: an overview of circulating and disseminated tumour cells. Clin Exp Metastasis. 2016;33(6):521-50.

28. Siravegna G, Bardelli A. Minimal residual disease in breast cancer: in blood veritas. Clin Cancer Res. 2014a;20(10):2505-7.

29. Siravegna G, Bardelli A. Genotyping cell-free tumor DNA in the blood to detect residual disease and drug resistance. Genome Biol. 2014b;15(8):449.

30. Beaver JA, Jelovac D, Balukrishna S, et al. Detection of cancer DNA in plasma of patients with early-stage breast cancer. Clin Cancer Res. 2014;20(10):2643-50.

31. Garcia-Murillas I, Schiavon G, Weigelt B, $\mathrm{Ng} \mathrm{C}$, Hrebien S, Cutts RJ, Cheang M, Osin P, Nerurkar A, Kozarewa I, Garrido JA, Dowsett M, Reis-Filho JS, Smith IE, Turner NC. Mutation tracking in circulating tumor DNA predicts relapse in early breast cancer. Sci Transl Med. 2015 Aug 26;7(302):302ra133.

32. Riva F, Bidard FC, Houy A, et al. Patient-specific circulating tumor DNA detection during neoadjuvant chemotherapy in triple-negative breast cancer. Clin Chem. 2017 Mar;63(3):691-9.

33. Agelaki S, Dragolia M, Markonanolaki $\mathrm{H}$, et al. Phenotypic characterization of circulating tumor cells in triple negative breast cancer patients. Oncotarget. 2017 Jan 17;8(3):5309-22.

34. Bidard FC, Peeters DJ, Fehm T, et al. Clinical validity of circulating tumour cells in patients with metastatic breast cancer: a pooled analysis of individual patient data. Lancet Oncol. 2014;15(4):406-14. 